\title{
SOME RECENT TRENDS AND DEVELOPMENTS IN DERMATOLOGY
}

\author{
G. Holti, M.D., and John T. INGRAM, M.D., F.R.C.P. \\ Department of Dermatology, Royal Victoria Infirmary, Newcastle upon Tyne
}

With the advance of science into the field of medicine, dermatology cannot be an isolated specialty and must be interpreted in terms of general medicine, physiology and pathology.

The trends and achievements in dermatology in post-war years come not only or chiefly from dermatologists, but from those engaged in the basic sciences and in general medicine and from teams comprising workers in all these fields. Much fundamental work on the physiology and biochemistry of skin has come from American schools, but in this country there have been valuable contributions, especially on the innervation of the skin, on vascular reactions, on tissue culture and electron microscopy.

Dermatologists are still without a satisfactory answer to a number of common, chronic and often distressing disorders, some of which seem protean in their manifestations, like erythema multiforme. Others, like psoriasis, are fixed reaction patterns by which susceptible individuals react to a multitude of internal or external stimuli. While recent improvements in conventional techniques have brought notable advances in the investigation of skin diseases, the study of functional changes by experimental methods seems to hold special promise. $8,9,14,16,17,24,28,42$

Investigative work on the skin fats of the surface film has stressed the importance of this film in relation to protection from injury and infection. ${ }^{7,41}$ It has important application in the field of industrial dermatoses, indicating the vulnerable workers and placing protective and therapeutic measures on a more rational plane. The integrity of the horny layer and of the deeper layers of the epidermis, which control permeability and absorption, is dependent in large part upon this surface film.

\section{Biochemical Lesions}

Inborn errors of the metabolism of fats, porphyrins or phenylalanine (to name only a few examples) may all have conspicuous cutaneous manifestations. In spite of their low incidence, these disorders have great fascination for the in- $\vec{\omega}$ vestigator because their study often sheds light $\bar{C}$ upon important unsolved problems of physiology 3 and pathology. These inborn disorders, as well as acquired deficiency states, may be classified as ' biochemical lesions ', to use Sir Rudolph Peters' ${ }^{35} \stackrel{\vec{A}}{\vec{A}}$ apt term. Such lesions can sometimes only be î demonstrated by experimental biological methods of of considerable complexity. The study of tissue 을 respiration in micro-respirometers, based on modi- fications of the now classical Warburg technique, $\frac{D}{O}$ holds special promise for dermatological research. Cruickshank $^{8}$ designed a comparatively simple and $\overrightarrow{\mathscr{\varphi}}$ inexpensive instrument of great precision which permits the continuous observation of the respiris tion and metabolism of minute skin slices for periods up to three days, during which time gas mixtures and liquids can be added to the cultures without disturbing the continuity of the experiment. Although this micro-respirometer was $\stackrel{\mathbb{Q}}{2}$ developed ' as part of a fundamental study of the $\overrightarrow{\overrightarrow{0}}$ ways in which skin is damaged by various toxic 3 substances and radiations ', it is also of great potential value in the study of normal skin metabolism and skin disorders due to a 'biochemical lesion'.

For some years dermatologists have suspected that a biochemical fault may be responsible for baffling disorders like psoriasis. A deficiency state may, in fact, be relative and only show itself as an impairment of adaptation to any type of stress from within' or without.

\section{The Mechanism of Pruritus}

Pruritus is the most important symptom in o dermatology. Until recently it had been considered $\mathcal{N}_{N}$ a variant of cutaneous pain largely because both $\omega$ pain and itch share the same sensory pathway throughout the peripheral and central nervous $\stackrel{0}{?}$

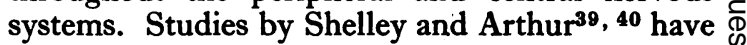
led to an entirely new concept of the mechanism of pruritus. Their initial observations were made on cowhage which had been used as itch powder $\stackrel{\vec{D}}{\mathbb{D}}$ for centuries. They identified the active com- 
pound responsible for pruritus as a proteinase, which, when introduced in moderate amounts into the skin, produced, after a latent period of a few seconds, intense pruritus without any concomitant visible changes. Larger amounts produced also erythema and urticaria. Similarly active proteinases were isolated from a variety of plants, bacteria, fungi and animals. Only endopeptidases produced itching; exopeptidases, which break down polypeptides, had no such effect, neither had endopeptidases such as pepsin, which are inactive in the neutral $p \mathrm{H}$ range. The intensity of the pruritus varied with different proteinases and was proportional to the quantity of enzyme introduced. Enzymes concerned with carbohydrate and lipid metabolism did not cause pruritus when pricked into skin. It is not yet certain whether proteinases produce itching by directly affecting nerve endings or by damaging epidermal cells. Epidermal cells are known to contain cathepsins, which are normally bound and inactive, but are released and activated after cellular injury. Plasmin, an important proteinase present normally in serum in an inactive form, is released in an active state in the skin during inflammation and has been demonstrated with other proteinases in allergic states. Shelley and Arthur believe that sustained pruritus often indicates an allergic reaction or the presence of chemicals of the proteinase-histamine liberator group.

\section{Urticaria}

This common affection has some particular facets, our knowledge of which has been extended in recent years. The type of urticaria provoked by heat, exertion and emotion in adolescents, characterized by a small weal with an intense flare and chiefly affecting the trunk and proximal parts of the limbs, is described as cholinergic urticaria. ${ }^{20}$ It is associated with the release of acetylcholine and, though the ultimate reaction is dependent upon the release of histamine, the condition is not responsive to antihistamines. It is not readily responsive to anti-cholinergic drugs, though they may inhibit the reaction for a limited period. In a number of patients with this affection it has been shown that the level of cholinesterase in the serum is markedly depressed, and it has been suggested that this favours the excess of acetylcholine which determines the reaction. ${ }^{33}$ Another suggestion is that the reaction, like that of miliaria, is dependent upon sweat retention. To avoid this, treatment by exfoliating the skin by exposure to ultra-violet irradiation has been reported as being successful. ${ }^{15}$

In children a common eruption is papular urticaria, which in large part corresponds to the normal type of urticaria in the adult. A number of workers have shown that the condition is frequently dependent upon an allergic sensitiveness in these children to the bites of fleas from cats, dogs and birds. Treatment of the animals, and of the patient, with a 2 per cent. DDT emulsion will effect a cure. ${ }^{6}$

\section{Fungus Infections}

In the range of fungal infections the antibiotic nystatin has proved valuable in the local treatment of candida albicans infection. It is applied as an ointment or may be used as pessaries or trochees. The administration of nystatin by mouth, $\mathrm{I}$ million units daily, though it is not absorbed, is an important part of treatment in many cases because it eradicates candida infection in the bowel.

Hypersensitivity to candida may be responsible for localized or generalized pruritus or urticaria and is treated on similar lines. These affections have come into greater prominence consequent upon the use of antibiotics and of steroid therapy.

The discovery by Gentles ${ }^{18}$ that the antibiotic griseofulvin was effective in controlling most ringworm infections is likely to revolutionize treatment, particularly of such intractable diseases as trichophyton rubrum infection of skin and nails and small-spore ringworm of scalp in children. The griseofulvin renders keratin resistant to these fungi and has an adverse influence upon the fungus itself, producing the so-called 'curling' effect. Though a cytotoxic drug when given in large doses intravenously to animals, no serious effects have been reported in man from its use, though temporary depression of the white cell count has been observed. ${ }^{3}$

Scalp ringworm in children is now well under control, but foot ringworm has increased considerably, especially in those industrial fields where shower baths have been installed for the use of workers. The problem threatened to be a formidable one, but may now yield to griseo fulvin. ${ }^{23,3}$

\section{The Investigation of Skin Disorders Attributed to Allergy}

Among the most challenging and tantalizing subjects in dermatology are the cutaneous manifestations of allergic sensitivity.

A clinical observation by Ramirez ${ }^{36}$ led to the discovery that at least certain types of protein sensitivity can be transferred with the serum of sensitized subjects. Loveless ${ }^{31}$ showed experimentally that sensitivity induced by the transfer of sensitized serum affected at least the skin and accessible mucous membrane, being fully established within 24 hours. The local transfer of sensitivity to normal skin by an intradermal injection, the Prausnitz-Küstner technique, has 
assumed practical as well as theoretical importance, although its application is limited by the very real danger of transmitting serum jaundice. It can also be applied to the quantitative assessment of sensitizing antibodies circulating in the plasma of an asthmatic, hay fever or urticaria patient.

The other main type of protein sensitivity reaction, elicited in its classical form by the tuberculin-trichophyton group, has been the object of many experimental investigations. Rich, ${ }^{47}$ in a comprehensive account, related how sensitization of the tuberculin type is widespread throughout the body. Practically every tissue has been claimed to have shown evidence of sensitization when tested experimentally, but not all claims have been confirmed. Reactions to protein sensitization have been studied largely in the skin, which is increasingly becoming a convenient indicator for the presence of a generalized state of sensitization by means of specific skin tests. $5,12,13,21,37,42$ There can be but little doubt that with such diagnostic or experimental tests we elicit reactions which also occur spontaneously and conversely it may be that disorders apparently confined to the skin and of unknown causation are merely the outward signs of generalized sensitization.

The number of reports dealing with clinical appearances due to suspected or inferred sensitization reactions is growing daily, but in order to be acceptable any such claim should be supported by sound evidence obtained by scientific experiments.

Almost any known type of rash has been observed as side effect of treatment with one or other of an ever-increasing number of drugs. Often the patient concerned is, or has been, receiving a number of different drugs simultaneously and the problem arises as to which of these drugs, if not the disease itself, has given rise to the eruption. Happily, drug rashes are often only trivial and transient, but at times it is necessary to consider carefully the risk of continuing treatment with a drug suspected of giving rise to side effects.

It is important to differentiate, if possible, between untoward reactions due to a direct toxic effect and allergic manifestations. Toxic effects will vary little from person to person and will be closely related to dosage while hypersensitivity, the result of an immunological process, shows much individual variation of the reaction pattern and is often induced by very small amounts of the sensitizing compound. Although rashes occurring during treatment with drugs and subsiding after such treatment has stopped are usually attributed to hypersensitivity to the drugs concerned, the skin lesions may, in fact, be due to other mechanisms such as vitamin deficiencies, enzymeinhibition by heavy metals and other metabolic upsets, or Shwartzman reactions. The only certain way to distinguish between allergic reactions and non-immunological toxic reactions is the identification both of the causal agent and its modus operandi. In spite of much work in this field during recent years we still lack an easy and reliable method of demonstrating allergy to drugs by skin-testing or immunological examination of the patient's serum or cellular blood constituents. ${ }^{1}, 22,28,42$

When skin testing, it is not always easy to differentiate between the nonspecific reaction of skin which has become unduly sensitive to even mild irritants and a truly specific allergic response. Severe local or systemic reactions during skin tests can be avoided by using initially very small test doses, for instance, in the case of suspected allergy to penicillin, not more than 50 units. Skin tests may be negative in the presence of true hypersensitivity because not enough allergen had been introduced or because the drug has to be metabolized to the sensitizing compound. In reports of negative or positive results of skin tests with drugs the method of testing and the quantity used should always be stated. Serological tests in suspected drug allergy include the search for specific antibodies against the drugs themselves and against their conjugates with proteins using a number of recently described tech $\leq$ niques $1,2,4,34,42$ If drug eruptions resemble the lesions of the delayed type of hypersensitivity with eczematous or nodular inflammatory infiltrations of the tuberculin-trichophyton type, immunological study of cells belonging to the lymphoreticular system may be rewarding. ${ }^{27}, 28,42$ Unfortunately a great deal of work still has to be done before such immunological techniques can become a routine procedure in hospital laboratories.

Yet a precise diagnosis of dermatological side effects during drug therapy is important because specific desensitization is often possible and occasionally necessary as with streptomycin allergy in some tuberculous patients. Furthermore, the skin lesions may be the only overt manifestation of an immunological process involving severely, but less obviously, other organs especially the liver, the kidneys, and the bone marrow.

\section{Patch Testing}

Irritant dermatitis and allergic reactions to $\stackrel{N}{N}$ external agents have inevitably increased with the $\mathrm{W}$ greater complexity of synthetic products. The industrial worker and the housewife are the important victims in this case, but a wide range of $\mathbb{D}$ reactions may also result from cosmetics and from garments worn next to the skin. ${ }^{41}$

The patch test is a simple and useful investigation to determine possible allergic reactions. ${ }^{5}$ 
The agent suspected of causing sensitization is applied suitably diluted to the unaffected skin, usually for a period of 48 hours. A positive reaction is one resembling the original dermatitis. The interpretation of patch tests requires experience and the procedure is not devoid of hazards.

\section{Tests for Light Sensitivity}

A method of identifying the so-called action spectrum for skin lesions in clinically light sensitive patients has been described by Magnus, Porter and Rimington. ${ }^{32}$ These workers used a quartz-monochromator with a high pressure Xenon arc as a light source which emits a continuous spectrum from the ultraviolet zone to the infra-red region. Narrow wave bands can be isolated and their effect upon the skin can be measured.

\section{Vascular Responses in Human Skin}

An important development in the study of vascular responses in the skin was the introduction by Lombard in 1912 of direct microscopical observation of blood vessels in intàct human skin. ${ }^{30}$ Sir Thomas Lewis and his colleagues using brilliantly simple experimental methods, defined the nature and time relations of the vascular reaction in the skin to various kinds of damage. Turning to specific clinical conditions they showed that Raynaud's phenomenon and acrocyanosis which had been attributed to disorders of vasomotor nerves were due to a local fault respectively in the digital arteries and dermal arterioles. Lewis and Grant $^{29}$ demonstrated 'refractoriness', i.e. the complete or partial absence of wealing as long as any erythema persisted. Earlier Ebbecke 11 had described in less precise terms how skin which had been subjected to manifold but mild injurious stimuli for several weeks became liable to show an eczematous reaction and that healing of small accidental abrasions seemed slower in this area. He further gained the impression that apparently recovered skin remained hyperaesthetic for some weeks or even months. Macroscopical or microscopical examination of the skin concerned did not produce any structural evidence for such abnormal behaviour.

As the skin allows continuous follow-up studies of indefinite duration and the easy application of scientific instruments without unduly interfering with natural conditions, some of these observations have been extended recently by assessing the rate of recovery of vascular function at the site of experimental injuries. ${ }^{25}$ For this purpose accurate measurements of skin temperature ${ }^{24}$ were used as an index of blood flow and estimates of wealing capacity were made in order to detect changes in capillary permeability. During this work the 'triple response' to histamine, representing as $\stackrel{\mathbb{Q}}{\stackrel{2}{2}}$ it were a practice mobilization of the immediate, $\subseteq$ mainly vascular, injury response, proved itself to be $\Rightarrow$ a simple yet most valuable clinical test, capable of $\stackrel{5}{\stackrel{5}{~}}$ producing quantitative results under standardized $\bar{C}$ conditions. It was observed that skin which had $\frac{\bar{F}}{\overline{0}}$ been strongly wealed by histamine or which had $\frac{\bar{C}}{\vec{T}}$ reacted intensely to ultra-violet irradiation showed $\stackrel{\Phi}{\circ}$ two long-lasting vascular abnormalities:- के

I. The minute vessels showed an increased $\overrightarrow{0}$ capacity to dilate in response to locally or reflexly induced vasodilation and a decreased $\vec{\omega}$ ability to contract in response to constrictor stimuli such as adrenalin or immersion in $\frac{0}{7}$ cold water.

2. Wealing to histamine pricks or to the applica- ô tion of a histamine liberator was reduced.

After large histamine reactions these vascular $\overrightarrow{\vec{A}}$ abnormalities could be demonstrated at the weal o site for three to eight weeks and after moderately severe ultra-violet reactions for six to 30 months. Such findings may be relevant to the common observation that in patients suffering from chronic skin diseases, often the same skin areas are affected in recurrent attacks, although during long res missions the skin may appear quite normal.

With similar methods vascular function was investigated in the skin of patients suffering from cutaneous manifestations of porphyria. ${ }^{26}$ It was found that in skin habitually exposed to light, but normal in appearance, both vasodilation and wealing were grossly impaired, while normal reactions were present on other body areas. This abnormal vascular behaviour may, at least partly, explain the poor inflammatory response observed in this condition and the increased vulnerability, even to very minor trauma, in habitually exposed skin. During that investigation the histamine prick, applied as a quantitative test, was of great help not only in defining site to site variations of vascular function in the skin, but also in assessing the severity of the condition.

Measurements of the disappearance rate of intradermally injected radioactive sodium have recently been used to study dermal reactions and especially circulatory changes. ${ }^{14}$ This method may yield information not obtainable by more conventional techniques.

\section{Vasculitis}

An increasing range of eruptions dependent upon ' allergic vasculitis' has been reported in recent years and in many cases the affection may be limited to the skin, though similar reactions are seen with systemic vascular disease.

Capillaritis, producing a bronzed discoloration 
of the skin from haemosiderin, associated with fine punctate and linear haemorrhages, is sometimes dependent upon drug allergy, as to Sedormid, ${ }^{1,10,38}$ carbromal, barbiturates and aspirin.

A livedo of larger pattern, often with erythematous patches, and painful small or large nodular lesions which may ulcerate, is the cutaneous picture produced by polyarteritis nodosa. This may be limited to the skin in some patients and remain so for years or throughout its course, though systemic involvement must always be considered.

Various patterns of obliterative vasculitis of smaller vessels, have been described, but the exact significance of these disorders is not clear. Again, they have been described as affecting skin alone or sometimes involving skin and intestinal mucosa only, these latter cases proving fatal from intestinal perforation. ${ }^{10,27 a}$

Among many other problems engaging attention, but still far from being elucidated, are the relationships of chronic discoid lupus erythematosus to systemic lupus erythematosus and of localized scleroderma (morphoea) and Raynaud's disease to systemic sclerosis. The wide group of mast cell disorders, dermatological and systemic, and the dermatological manifestations of carcinoid tumour have received attention.

Psoriasis, arthritis, Reiter's syndrome and pustular psoriatic eruptions all have some common features that demand investigation.

Grace and Dao, ${ }^{19}$ using the passive transfer technique, have recently demonstrated, in a patient with dermatomyositis with breast carcinoma, hypersensitivity to an extract of the tumour. They suggest that other patients with dermatomyositis and coexistent tumours 'should be studied similarly to determine if this mechanism accounts for the frequent association of cancer with this " collagen disorder" '.

\section{REFERENCES}

1. ACKROYD, J. F. (1954), Clin. Sci., 13, 409.

2. ACKROYD, J. F. (1955), Sang, 26, 115.

3. BARLOW, A. J. E., CHATTAWAY, F. W., HARGREAVES, G. K., and LA TỎUCHE, C. J. (1959), Brit. med. F., il, r 141 r.
4. BENACERRAF, B., and GELL, P. G. H. (1959), F. Immunol., 2, 53 .

5. BETT, D. C. G., and CALNAN, C. D. (1957), Tr. St. Fohn's Hosp. derm. Soc. (Lond.), 39, 20.

6. BOLAM, R. M., and BURTT, E. T. (1956), Brit. med. $\mathcal{f}$. i, 1130 .

7. BOUGHTON, B., and WHEATLEY, V. R. (r959), $\mathcal{F}$. invest. Derm., 33, 49 .

8. CRUICKSHANK, C. N. D. (1954), Exp. Cell. Res., 7, 374.

9. CRUICKSHANK, C. N. D., COOPER, J. R., and CONRAM, M. B. (1959), Ibid., 16, 695 .

ro. DEGOS, R. (1952), Bull. Soc. Dermat. et Syph., 6r, 211.

r. EBBECKE, U. (1917), Pflug. Arch. ges. Physiol., 169, x.

12. EPSTEIN, W. L., and KLIGMAN, A. M. (1957), F. invest . Derm., 28, 29i.

13. EPSTEIN, W. L., and KLIGMAN, A. M. (1959), Ibid., 33, 23 r.

I4. FAIRBURN, E. A. (1959), Ibid., 32, 95.

I5. GARRETTS, M. (1958), Brit. F. Derm., 70, I66.

16. GELL, P. G. H. (1957), F. clin. Path., 10, 67.

17. GELL, P. G. H., and BENACERRAF, B. (1959), f. Immunol., 2, 64 .

i8. GENTLES, J. C. (r959), Brit. F. Derm., 71, 427.

19. GRACE, J. T., and DAO, Th. L. (1959), Cancer, 12, 648.

20. GRANT, R. T., PEARSON, R. S. B., and COMEAU, W. J. (1936), Clin. Sci., 2, 253 .

21. HARDWICKE, J., SOOTHILL, J. F., SQUIRE, J. R., and HOLTI, G. (1959), Lancet, i, 500 .

22. HOIGNE, R., GRASSMANN, W., and STORCK, H. (1955), Schweiz. med. Wschr., 85, 578 .

23. HOLMES, J. C., and GENTLES, G. C. (1956), Lancet, ii, 62. 24. HOLTI, G. (1955), Clin. Sci., 14, 137.

25. HOLTI, G. (I955), Ibid., 14, 143.

26. HOLTI, G., RIMINGTON, R., TATE, B.C., and THOMAS, G. (1958), Quart. F. Med., New Series, 27, I.

27. INDERBITZIN, T. (1956), Int. Arch. All., 8, 150.

27a. INGRAM, J. T. (1957), A.M.A. Arch. Derm., 75, 820.

28. LAWRENCE, H. S. (I959), 'The Transfer of Hypersensitivity of the Delayed Type in Man, Cellular and Humoral Aspects of the Hypersensitive States.' Edited by H. S. Lawrence, New York, Hoeber-Harper, 1959.

29. LEWIS, T. (1927), "The Blood Vessels of the Human Skin and their Responses.' London: Shaw and Sons, Ltd.

30. LOMBARD, W. P. (rgr 2), Amer. F. Physiol., xxix, 335.

31. LOVELESS, M. H. (194I), f. Immunol., 41, I5.

32. MAGNUS, I. A., PORTER, A. D., and RIMINGTON, C. (1959), Lancet, i, 91 2.

33. MORGAN, J. K. (1953), f. invest. Derm., 21, 173.

34. OUCHTERLONY, O. (1948), Acta path. microbiol. scand., 25, 186.

35. PETERS, R. A. (1945), Brit. med. Bull., 3, 81.

36. RAMIREZ, M. A. (1919), F. Amer. Med. Ass., 73, 984.

37. RICH, A. R. (I944), 'The Pathogenesis of Tuberculosis,' Springfield, Illinois.

38. RUITER, M. (1954), Brit. F. Derm., 66, 174.

39. SHELLEY, W. B., and ARTHUR, R. P. (1955), Excepta Medica, Sec. XIII, Dermatol. and Venereol., 9, I71.

40. SHELLEY, W. B., and ARTHUR, R. P. (I955), A.M.A. Arch. Derm., 72, 399.

4I. SQUIRE, J. R., CRUICKSHANK, C. N. D., and TOPLEY, E. (1950), Brit. med. Bull., 7, 28.

42. SQUIRE, J. R. (1955), 'Laboratory and Clinical Findings in Hypersensitivity.' Lectures on the Scientific Basis of Medicine IV, $1954-1955$. 